\title{
Effect of 2,4-D pre-treatment on quality during ripening of on-tree longan fruit
}

\author{
Yunfen Liu ${ }^{1}$, Lingyan Liao ${ }^{1}$, Feilong Yin ${ }^{1}$, Boyang Guan ${ }^{1}$, Hongmei Pu$^{2}$, Chao Kang ${ }^{1}$, and Liang Shuai ${ }^{1, *}$ \\ ${ }^{1}$ College of Food and Biological Engineering/Institute of Food Science and Engineering Technology, Hezhou University, Hezhou 542899, \\ China \\ ${ }^{2}$ Agro-food Science and Technology Research Institute, Yunnan Academy of Agricultural Sciences, Kunming 650205, Yunnan, China
}

\begin{abstract}
The aim of this study was to investigate the effect of $20 \mathrm{mg} / \mathrm{L} \mathrm{2,4-D}$ on the quality during ripening of on-tree longan fruits. We investigated external and internal properties of the on-tree longan fruits. The results showed that 2,4-D treatment promotes the growth based on the fruit size and weight. The respiration rate, contents of TSS, total soluble sugar, sucrose, glucose, fructose, and hexose revealed an increasing tendency with advancing the maturity and reached the high status during 110-126DPA. By contrast, the relative electric conductivity and TA content displayed a declining trend during the ripening stage, increase with the senescence. All these pieces of information indicated that 2,4-D treatment could effectively promote the sensory quality of on-tree longan fruit, prolong the harvest time to 118DPA, while CK should harvest before 110DPA.
\end{abstract}

\section{Introduction}

Longan (Dimocarpus longan Lour.) is a kind of tropical and subtropical fruit with high business value, it has been cultivated widely in many countries, especially in China, and other Asia-Pacific areas ${ }^{[1-3]}$. It contains rich nutrients and many other pharmacological materials ${ }^{[4]}$. Among that sugars play a vital role in fruit quality and are considered as a signal molecule during fruit growth and development which are determined by sugar component and content ${ }^{[5,6]}$. Sugar of longan fruit mainly consists of sucrose, fructose and glucose with different ratios at different stages ${ }^{[7]}$. Previous studies have shown that longan fruit sugar receding may occur at unreasonable harvest time, resulting in a change of sugar composition ${ }^{[8]}$, contents of total soluble solids and titratable acidity increase during fruit development, and this is related to the content of sugar ${ }^{[9-11]}$, thus control sugar receding is an important subject to preserve the quality of longan fruit.

Moreover, there are a series of physical and biochemical changes during fruit development which include fruit size and shape and some other alternations inside the longan fruit, for instance, respiration rate, relative electric conductivity and Malondialdehyde (MDA), these factors are also important assessment indicators of fruit quality and shelf-life ${ }^{[9-11]}$. The diameter in ripening longan fruit is about $15-30 \mathrm{~mm}$, and as a non-climacteric fruit will no longer ripen once harvest, then rapidly deteriorate ${ }^{[14]}$. Respiration will consume energy if harvest at the non-optimal time, decrease fruit weight, and accelerate senescence ${ }^{[15]}$, meanwhile, during fruit development and ripening stage, cell membrane permeability has changed due to many secondary metabolites produced ${ }^{[10]}$. Therefore, it is necessary to choose an appropriate harvest time.

It is known that phytohormones are involved throughout the growth and development of plant ${ }^{[16-18]}$. Some studies have pointed out that auxin plays a significant role in fruit development and ripening ${ }^{[19-21]}$. 2,4-dichloro phenoxy acid (2,4-D) is a common auxin that could increase fruit size and its effect concentration and date depends on the species ${ }^{[22]}, 2,4-\mathrm{D}$ treatment also could reduce the fruit drop of navel orange ${ }^{[23]}$. However, it is no more evidence the effect of 2,4-D treatment on the quality of on-tree fruit.

In this work, longan fruit, Dimocarpus longan Lour. cv. Baihuamu was chosen as the material because of its long sugar receding periods to assess the development and quality after 2,4-D treatment before harvesting. It is significant for a better understanding of the effect of 2,4$\mathrm{D}$, guiding the optimal harvest time.

\section{Materials and methods}

\subsection{Materials and treatments}

8-10 years old longan(Dimocarpus longan Lour. cv. Baihuamu) trees with consistent growth and moderate vigor were selected to sprayed 2,4Dichlorophenoxyacetic acid (2,4-D) before harvesting from an orchard in the Institute of Fruit Tree Research in Guangdong Academy of Agricultural Sciences, Guangzhou, Guangdong Province, China. The spraying time was after full-bloom stage 60 days and 80 days for twice, and the concentration of 2,4-D was $20 \mathrm{mg} / \mathrm{kg}$, 
while the distilled water was control. Three biological replicates were analyzed for each treatment.

Fruit samples were taken after 94 days,102 days(ripening stage),110 days(full-ripening stage),118 days, 126 days and 134 days post-anthesis(DPA), more than 50 fruits were harvested from different canopy position. Then transported to the laboratory within $4 \mathrm{~h}$, cut off the stem, fruits with uniformity of maturity, shape and color, and without any diseases and blemishes were selected as materials. Pulps were immediately frozen in liquid nitrogen and stored at $-80^{\circ} \mathrm{C}$.

\subsection{Biological characteristics}

Biological characteristics were measured on 30 fruits of each time point. Long diameter, short transverse diameter and vertical diameter were measured by a digital vernier caliper with a precision of $0.01 \mathrm{~mm}$, the analyses were conducted twice. Weight was determined by an electrical scale with a precision of $0.01 \mathrm{~g}$, the analyses were taken 5 times.

\subsection{Extraction and assay of titratable acidity(TA), total soluble solids(TSS) and total soluble sugar}

Pulp from 20-30 fruits was juiced, then filtered through 4layer gauze. The filtrate was randomly distributed into 3 groups for analysis of TA, TSS and total soluble sugar. 1 $\mathrm{mL}$ of the solution was used to measure TA content by the method of titrating with $0.1 \mathrm{~mol} / \mathrm{L} \mathrm{NaOH}$. TSS was directly measured using a digital refractometer(PR-32 $\alpha$, ATAGO Co., Ltd., Tokyo, Japan). The content of total soluble sugar was determined according to the method of anthrone sulphuric acid ${ }^{[23]}$

\subsection{Measurement of membrane permeability in pericarp and respiration rate}

Membrane permeability was expressed as the relative electric conductivity with \% according to the method of Wang et al ${ }^{[25]}$ with small changes. 30 pericarp discs $(0.5 \mathrm{~mm}$ in diameter)from 10 each fruit, washed twice in deionized water, dried with filter paper then immersed in $25 \mathrm{~mL}$ distilled water for $30 \mathrm{~min}$. Initial conductivity was determined with the DDS-307 conductivity meter(Shanghai Scientific Instruments, Shanghai, China). Total conductivity was measured after boiling for $15 \mathrm{~min}$ and cooled to $25^{\circ} \mathrm{C}$. The relative electric conductivity was expressed as a percentage of the initial value of the total value.

The method of Wang et al ${ }^{[25]}$ was employed to determine the respiration rate with some modifications. 20 fruits were sealed for $2 \mathrm{~h}$ and then exhausted air $1 \mathrm{~mL}$, measured by meteorological chromatograph(GC-17A, SHIMADZU, Japan). Respiration rate was expressed as carbon dioxide released per kilogram per hour of fresh weight $\left(\mathrm{mg} \mathrm{CO}_{2} \cdot \mathrm{kg}^{-}\right.$ $\left.{ }^{1} \cdot \mathrm{h}^{-1}\right)$. The analyses were carried out for 3 times.

\subsection{HPLC analysis of sucrose, glucose and fructose}

The assay method of sugar component was reported by Luo et al ${ }^{[8]}$ with a small modification. $1 \mathrm{~g}$ pericarp samples were in a microwave oven for $30 \mathrm{~s}$ to deactivate the enzymes, and homogenized in a mortar with $2 \mathrm{~mL}$ ultrapure water, the homogenate was transferred into a centrifuge tube. The mortar and pestle were rewashed for 3-4 times, the mixture was diluted to $12 \mathrm{~mL}$. $2 \mathrm{~mL}$ aliquot was taken to centrifuge at $13000 \mathrm{rpm}$ for $15 \mathrm{~min}$ at ambient temperature. The supernatant was passed through a Sep-Pak $\mathrm{C}_{18}$ Cartridge (Water Corporation, Milford, Massachusetts, USA). Sugar was detected by high-performance liquid chromatography(HPLC) using an Agilent 1200 HPLC system(Agilent Technologies, Waldbronn, Germany) with a G1362A refractive index detector cell maintained at $40^{\circ} \mathrm{C}$. A transgenomic CARB sep Coregel 87C column (CHO-99-5860) With a guard column cartridge (Transgenomic CARB Sep Coregel $87 \mathrm{C}$ cartridge) was used. The column was maintained at $80^{\circ} \mathrm{C}$ with a thermostatted column compartment. The injection volume was $10 \mu \mathrm{L}$. Samples were eluted with double-distilled water. The flow rate was $0.6 \mathrm{~mL} \cdot \mathrm{min}^{-1}$. Quantification of sugars (sucrose, glucose and fructose)in samples was performed according to external standard solution calibrations (sugar reagents were purchased from Sigma Chemical Co.). The sugar content was calculated according to the peak area of samples and the standard curve of calibrators and expressed as $\mathrm{mg} \cdot \mathrm{g}^{-1} \mathrm{FW}$.

\subsection{Statistical analysis}

The variance of data was analyzed Using SPSS software package release 18.0(SPSS Inc. Chicago, IL, USA). The data were displayed as the means \pm standard error.

\section{Results and discussion}

\subsection{Effects of 2, 4-D pre-treatment on external character of on-tree longan fruit}

Longan fruit'Baihuamu' development and ripening occurs approximately $110 \mathrm{~d}$ after anthesis, the shape of fruits has changed during the time. Some external characters were monitored (Figure 1a,b,c). The transverse diameter(both long diameter and short transverse diameter) showed a tortuous growth trend in all treatments, and the treatment of 2,4-D was higher than $\mathrm{CK}$ (Figure 1a,b). It is notable that the transverse diameter value descended at $110 \mathrm{DPA}$. It may be the errors of sampling. Compared with the transverse diameter, the vertical diameter was increased as a smooth line (Figure1c), and during the period, a vertical diameter of 2,4-D treatment was higher than CK. This may because auxin can increase cell size ${ }^{[26]}$. During the development, fruit weight gradually increased, at the harvest stage, different cultivars weight various from 5 g-20 $\mathrm{g}^{[2]}$. Spraying with 2,4-D promoted the increasing trend, which higher than $\mathrm{CK}$ almost $1.261 \mathrm{~g}$ and $1.252 \mathrm{~g}$ at 102 
DPA and 110 DPA, respectively (Figure1d). These results indicated that 2,4-D could promote longan fruits size and weight. Similar results occurred in the research of auxin on loquat $^{[27]}$.
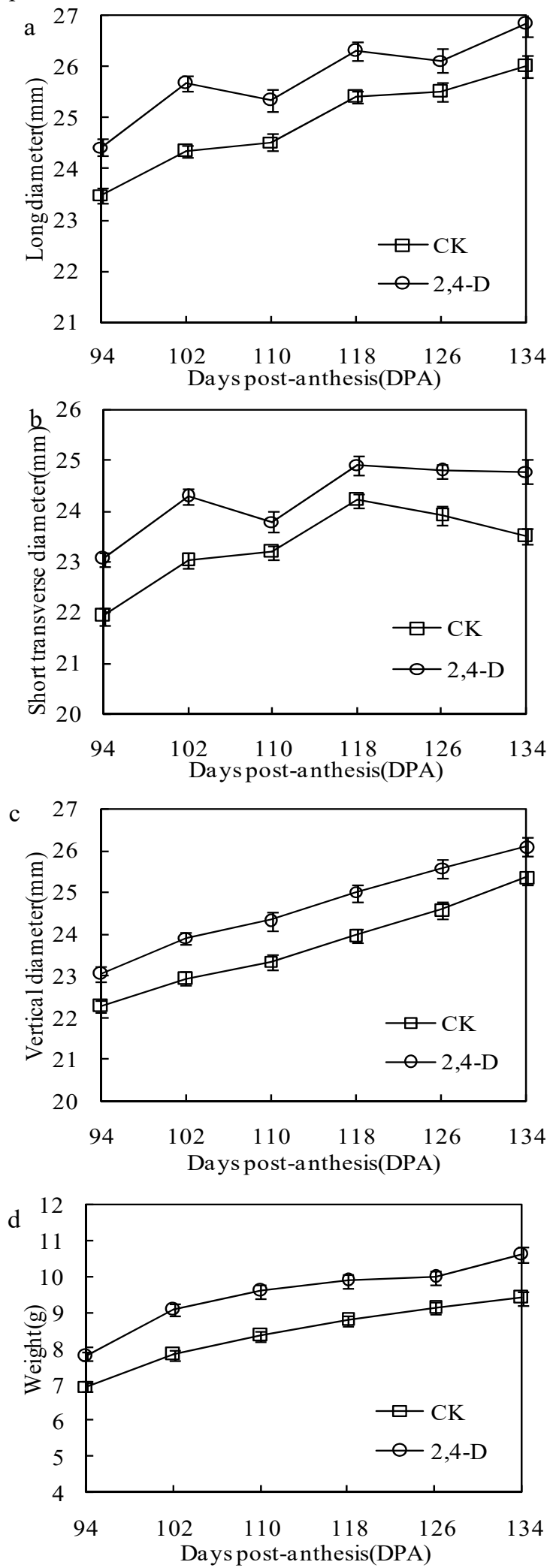

Fig.1. Effects of 2, 4-D pre-treatment on long diameter(a), short transverse diameter(b), vertical diameter(c) and weight(d) of ontree longan fruit

\subsection{Effects of 2, 4-D pre-treatment on content of TA, TSS and total soluble sugar of on-tree longan fruit}

The flavor indicators of fruit consist of TSS, total soluble sugar and $\mathrm{TA}^{[28]}$. As Figure 2a shown, the TA contents of both groups displayed a similar pattern: decline firstly and then rising. The 2,4-D treatment reached the lowest at $110 \mathrm{DPA}$, whereas CK reached the lowest before the ripening stage, and at the later stage of maturity, TA content of 2,4-D treatment was higher than CK. For the TSS contents of CK and 2,4-D treatment, the pattern was opposite to the TA content: increasing firstly then descent(Figure 2b). In comparison to the CK, 2,4-D treatment demonstrated a higher level of TSS content. Figure 2c showed that the total soluble sugar increased with increasing ripening time, after full-ripening time, the total soluble sugar exhibited a downward tendency; While compared with CK, 2,4-D treatment demonstrated a higher level. As figure $2 \mathrm{~d}$ shown, the tendency of 2,4-D treatment was increasing to $110 \mathrm{DPA}$ and then decreasing; while CK increasing before 102 DPA. Longan is a kind of non-climacteric fruit that will not continue to ripen once harvesting, therefore harvest on time is much important.TSS/TA ratio was applied to determine fruit maturity for most Chinese cultivators ${ }^{[14]}$. All results indicated that with the development of longan fruit, the flavor and quality gradually became worse, especially after full-ripening stage. 2,4-D treatment could maintain the flavor and prolong the on-tree fruit preservation.
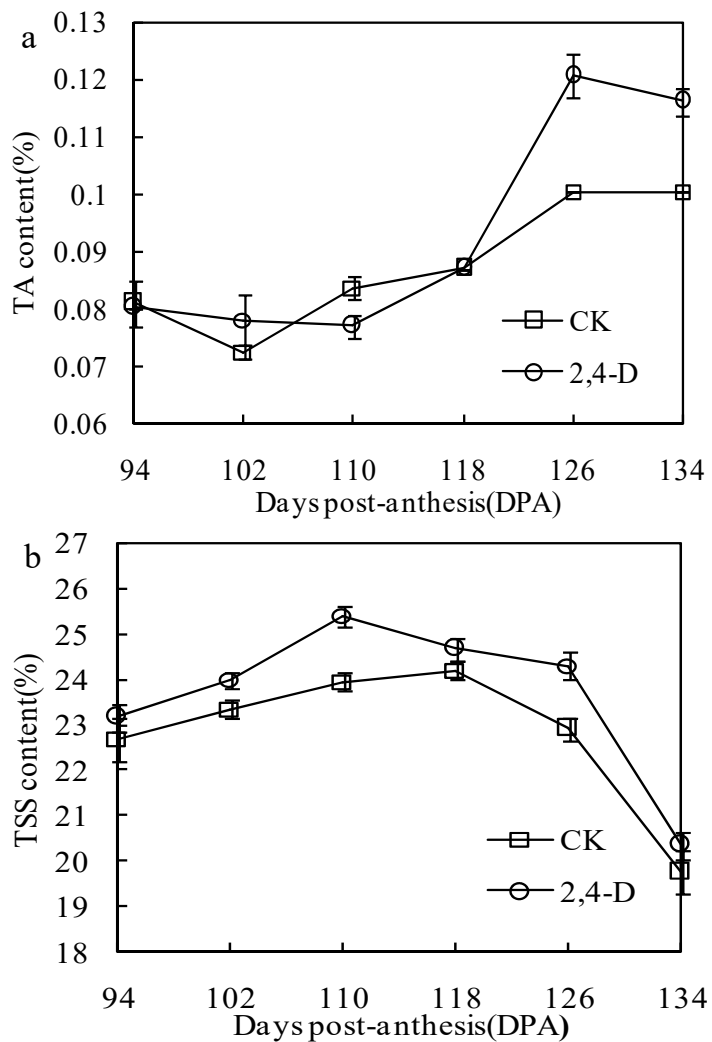

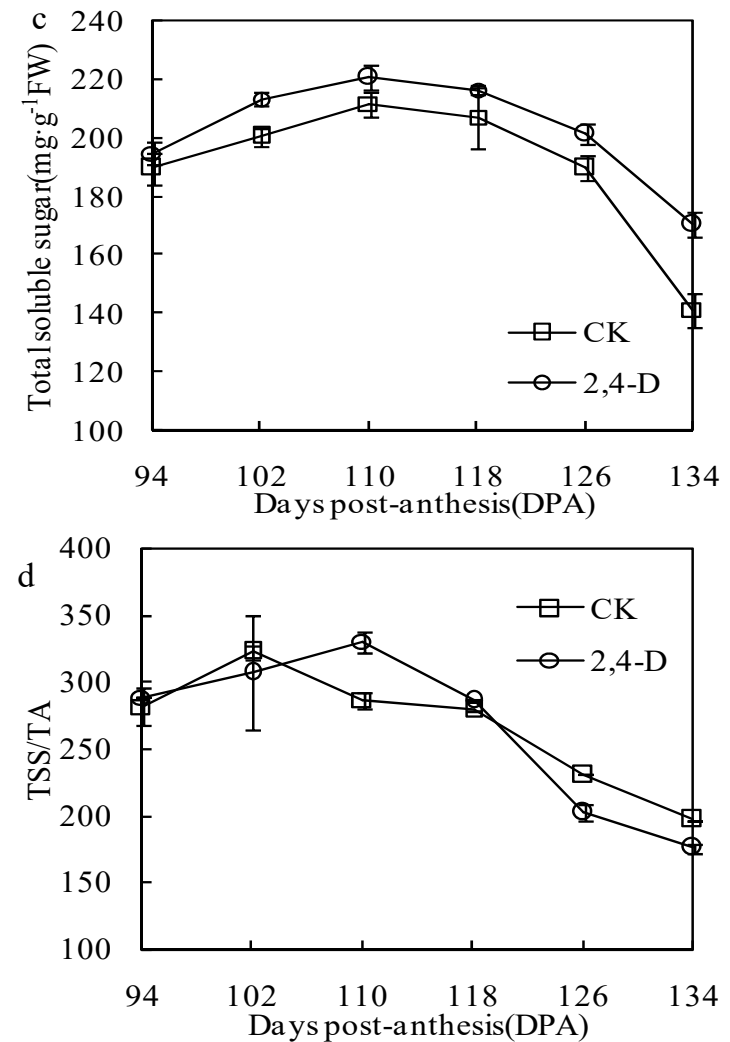

Fig.2. Effects of 2, 4-D pre-treatment on content of TA(a), TSS(b), total soluble sugar(c) and TSS/TA (d)of on-tree longan fruit

\subsection{Effects of 2, 4-D pre-treatment on membrane permeability and respiration rate of on-tree longan fruit}

Figure $3 \mathrm{a}$ illustrated that the relative electric conductivity in CK went up a little at 108 DPA, following by a sharp descent at 110 DPA, then a rapid rise occurred at 118 DPA, descended during the later stage of maturity. However, the relative electric conductivity of 2,4-D treatment showed a rapid descent at $110 \mathrm{DPA}$, then raised until $126 \mathrm{DPA}$, finally decreased. The 2,4-D treatment showed a lower level before and after the full-ripening stage. Auxin could promote fruit ripening and softening ${ }^{[29]}$, on the other hand, the fruit reaches maturity and enters senescence caused cell separation $^{[30]}$, all of which leads to higher permeability of 2,4-D treatment at the last maturity stage.

Respiration plays a vital role in the growth and development of plants, and it varies with different stages of fruit development ${ }^{[31]}$. As shown in Figure $3 b$, before fullripening (110 DPA), the fruit respiration rate was raised both in CK and 2,4-D treatment, then gradually decreased. However, the respiration rate of 2,4-D maintained a lower level. As we all know that respiration consumes organic sources resulting in fruit quality worse. Our study showed a lower respiration rate thus retaining the quality of on-tree fruit.
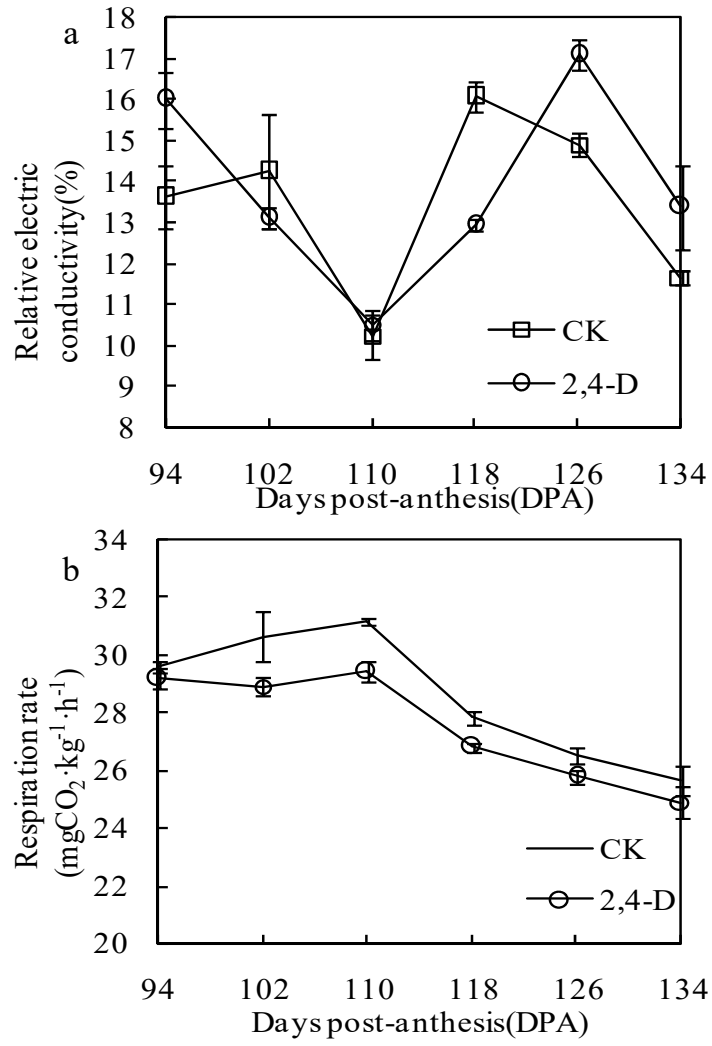

Fig.3. Effects of 2, 4-D pre-treatment on membrane permeability(a) and respiration rate(b) of on-tree longan fruit

\subsection{Effects of 2,4-D pre-treatment on sugar components of on-tree longan fruit}

Sugars are kinds of components determining the sensory quality, mainly consist of sucrose, glucose and fructose ${ }^{[32]}$. The sugar compositions and contents vary with fruit development as well as environmental condition ${ }^{[32,33]}$. As figure $4 \mathrm{a}$ shown, the sucrose content of the CK group increased before 110 DPA then dropped down, in comparison, the sucrose content of 2,4-D treatment ascended before 108DPA, then decreased after fullripening. In brief, a higher level of sucrose content revealed in 2,4-D treatment. For the glucose content, fructose content and hexose content, the CK and 2,4-D treatment displayed a similar tendency: decreased at 108 DPA, then ascended until 126 DPA, and finally decline; whereas the contents of 2,4-D treatment peaked at 118 DPA or 126 DPA, then descended, and maintained higher levels after 102 DPA(Figure $4 b, c, d)$. The ratio of sucrose/hexose of 2,4-D treatment decreased during the period of ripening, the ratio of $\mathrm{CK}$ increased firstly and then decreased, and remained at a high level all the time(Figure $4 \mathrm{e}$ ). In a word, before ripening, sucrose, glucose, fructose content of longan fruit increased, afterward decreased. Previous research showed that glucose and fructose contents had a great accumulation coincided with fruit ripening, and dropped in sucrose, the sucrose presented a maximum in the ripening fruit ${ }^{[34]}$. Sucrose is the major end product in photosynthesis and often hydrolyzed into glucose and fructose ${ }^{[35]}$. The decrease of sucrose caused the sugar receding in longan fruit which seriously affected the fruit quality ${ }^{[8]}$. 
Obviously choosing optimal harvest time is vitally important. In our results that 2,4-D retarded the descent of sucrose, increased the content of glucose and fructose, slowed down the sugar receding rate, maintained the fruit quality.
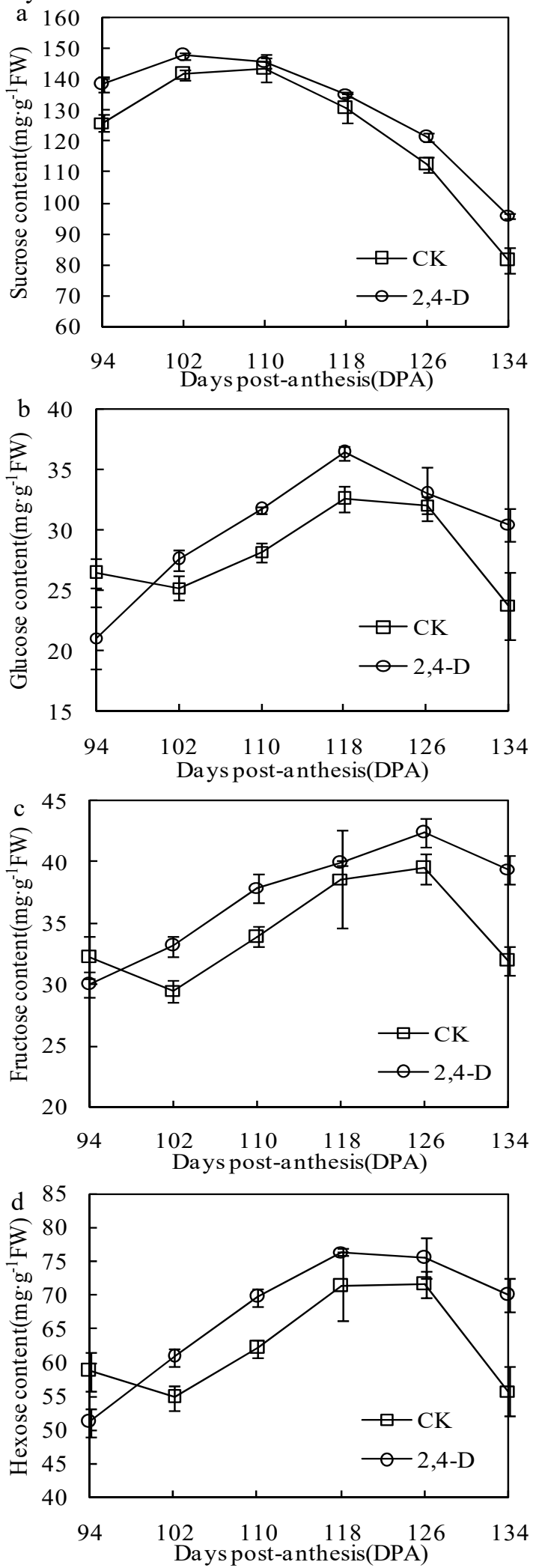

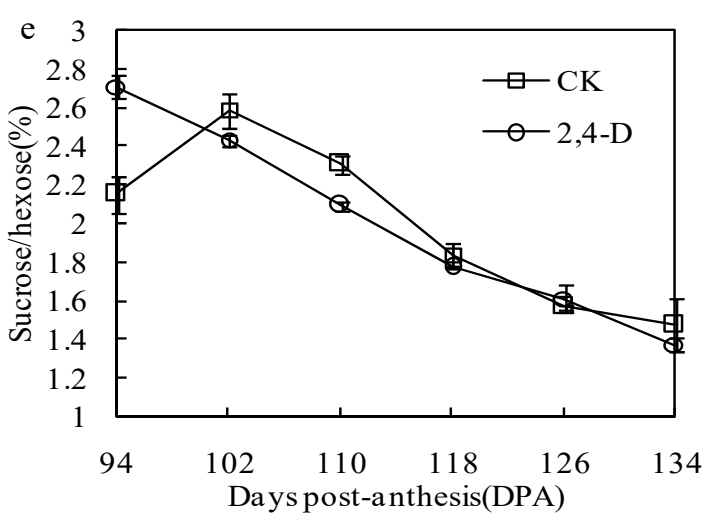

Fig.4. Effects of 2,4-D pre-treatment on sucrose content(a), glucose content(b), fructose content(c), hexose content(d) and ratio of sucrose/hexose(e)of on-tree longan fruit

\section{Conclusion}

In our study, we investigated the properties of 2,4-D treatment during the ripening of on-tree longan fruit. By measuring the appearance trait(size and weight) and some flavor indexes, it has demonstrated that 2,4-D treatment could promote the growth and effectively retain the sensory quality of on-tree longan fruit, prolong the harvest time to118DPA, while CK should harvest before 110DPA.

\section{Acknowledgments}

This work was supported by the Natural Science Foundation of China(31860457), the Natural Science Foundation of Guangxi Province of China(2019GXNSFBA245037), the Hezhou innovationDriven Development Project(PT1907006), Guangxi First-class Discipline (Cultivation) Project of Food Science and Engineering.

\section{Reference}

1. $\mathrm{Y} F \mathrm{~F}$ Lin, $\mathrm{Y} \mathrm{Z}$ Lin, $\mathrm{Y} \mathrm{X}$ Lin, et al.Carbohydrate Polymers, 217:126-134(2019).

2. S Y Shi, W Wang, L Q Liu, et al. Scientia Horticulturae, 207:160-167(2016).

3. Y F Lin, Y X Lin, H T Lin, et al. Food Chemistry, 225(15):31-36(2017).

4. Y F Lin, Y X Lin, H T Lin, et al. Food Chemistry, 240:863-869(2018).

5. T Luo, J J Niu, X M Guo, et al. Journal of the ence of Food and Agriculture, 99:1098-1107(2019).

6. S Iqbal, Ni X, M S Bilal, et al. Gene, 742(2020).

7. R E Pall, N J Chen. Hortscience, 22(6): 13031304(1987).

8. T Luo, L Shuai, L Y Liao, et al. Journal of Agricultural and Food Chemistry, 67(1): 352363(2018).

9. T Montero, E Mollá, R Esteban, et al. Scientia Hortic, 65(4): 239-250(1996). 
10. J Oustric, S Antoine, J Giannettini, et al. Acta Physiologiae Plantarum, 37(11), (2015).

11. L Shuai, L T Gu, W H Liu, et al. Chinese Journal of Tropical Crops, 37(10): 1900-1907(2016).

12. V Deshi, M W Siddiqui, F Homa, et al. Acta Physiologiae Plantarum, 42(5), (2020).

13. S Y Shi, W Wei, L Q Liu, et al. Journal of Food Engineering, 118(1): 125-131(2013).

14. Y M Jiang, Z Q Zhang, D C Joyce, et al. Postharvest Biology \& Technology, 26(3): 241-252(2002).

15. I Recasens, A Benavides, J Puy, et al. Journal of the Science of Food and Agriculture, 84(8): 765 771(2004).

16. $\mathrm{P}$ Davies. Plant Hormones and their Role in Plant Growth and Development, (1987).

17. M Saniewski, M Dziurka, K Dziurka, et al. Plant Growth Regulation, 91: 383-396(2020).

18. L Q Liu, J G Li, B Shu, et al. Southwest China Journal of Agricultural Sciences, 29(11): 2698-2703(2016).

19. M Satoshi, K Kaori, N Kaori, et al. Scientia Horticulturae, 241: 329-338(2018).

20. L Monsalve, A Ayala-Raso, M Bernales, et al. Data in Brief, 21: 1521-1525(2018).

21. I El-Sharkawy, S Sherif, T Qubbaj, et al. Postharvest Biology \& Technology, 112: 215-223(2016).

22. M Agustí, N Gariglio, A Castillo, et al. Plant Growth Regulation, 41(2): 129-132(2003).

23. X Wang, J Y Chen, S J Liu. Acta Horticulture Sinica, 39(3): 539-544(2012).

24. M Y Chen, $\mathrm{H}$ T Lin, S Zhang, et al. Food and Bioprocess Technology, 8(5): 971-982(2015).

25. H Wang, W Zhi, H Qu, et al. Chemistry Central Journal, 9(1), (2015).

26. R Stern, $M$ Flaishman, $\mathrm{R}$ Ben-Arie. Scientia Horticulturae, 112(3): 304-309(2007).

27. C Reig, C Mesejo, A Martínez-Fuentes, et al. Scientia Horticulturae, 210: 213-219(2016).

28. B D Liu, W X Jiao, B G Wang, et al. Scientia Horticulturae, 249: 100-109(2019).

29. W F Zeng, Z Q Wang, L Pan, et al. Journal of Fruit Science, 32(2): 200-205(2015).

30. P White. Journal of Experimental Botany, 53(377): 1995-2000(2002).

31. E Hernández-Montes, J Escalona, M Tomàs, et al. Physiologia Plantarum, (2020).

32. J Zheng, C H Huang, B R Yang, et al. Journal of Food Biochemistry, 43(6), (2018).

33. E Desnoues, M Génard, B Quilot-Turion, et al. Plant Journal, 94(4): 685-698(2018).

34. S Almansa, F Hernández, P Legua, et al. Journal of the Science of Food and Agriculture, 96(12): 40984105(2016).

35. H J Wan, L M Wu, Y J Yang, et al. Trends Plant Sci, 23(2): 163-177(2018). 